\title{
The Existence of a Critical Salt Concentration for Particle Release
}

\author{
K. C. KHILAR ${ }^{1}$ AND H. S. FOGLER \\ Department of Chemical Engineering, University of Michigan, Ann Arbor, Michigan 48109
}

Received September 30, 1981; accepted March 28, 1984

\begin{abstract}
A critical salt concentration (CSC) was found to exist in the water sensitivity of Berea sandstone. If the salinity of the permeating fluid falls below the CSC, the sandstone permeability is significantly reduced as a result of clay particles being released from the pore walls and blocking the pore throats. However, when changes in salinity occur above this threshold value, clay particles are not released from the pore walls in the sandstone and therefore no reduction in core permeability occurs. The CSC was determined by core flood experiments in conjunction with particle analysis of core effluent samples. The CSC exists only in the case of monovalent cations and is virtually nonexistent for cations having a valence greater than one. Even among monovalent cations the CSC varies considerably and it decreases with increasing ion exchange affinity of the clay for the counterion. For polyvalent counterions, the release of clay particles is effectively prevented due to the strong ion-exchange affinity of clay for the counterion. The critical salt concentration was also found to depend on the temperature, but not on the flowrate, of the electrolyte solution. The temperature dependence has been explained by using the Derjaguin-Landau-Verwey-Overbeek (DLVO) theory of stability of colloidal dispersions.
\end{abstract}

\section{INTRODUCTION}

The water sensitivity of sandstone, a problem in colloid chemistry that has been known to exist for the past 40 years, is a phenomenon whereby the permeability of the sandstones containing clay minerals decreases rapidly and significantly when fresh water replaces the salt water initially present in the sandstone. The results of a standard experiment to demonstrate this phenomenon which is known as "water sensitivity" are shown in Fig. 1. In this experiment a 1-in.-diameter core of Berea sandstone is vacuum saturated with a $3 \mathrm{wt} \%$ solution of $\mathrm{NaCl}$. The core is then placed in a core holder and a $3 \mathrm{wt} \%$ salt water solution is passed through the core in the axial direction. After a period of time the solution flowing through the core is abruptly switched from salt water to fresh water. One observes from Fig. 1 that the overall permeability of the core drops dramatically by a factor of about 100

\footnotetext{
${ }^{1}$ Currently Department of Chemical Engineering, IIT, Bombay, India.
}

after only one or two pore volumes of fresh water have entered the system. To see the effect of flow reversal, after a number of pore volumes of fresh water have entered the core, the direction of the flow is reversed so that fresh water now enters through the rear of the core. After a constant pressure drop is achieved, the flow is switched to salt water in the same (i.e., reverse) direction. Finally the direction of the flow is again reversed so that salt water enters the front of the core and flows through the core in the forward direction. The effects of these flow reversals is seen in Fig. 1, and the two most important features of this phenomenon are

1. The instantaneous but temporary permeability gain when the direction of fresh water flow through the core is reversed.

2. The permanent permeability gain when the direction of the salt water flow is reversed.

These observations are a manifestation of the peptization and flocculation of clay particles in the water sensitivity of Berea sandstones (1). 


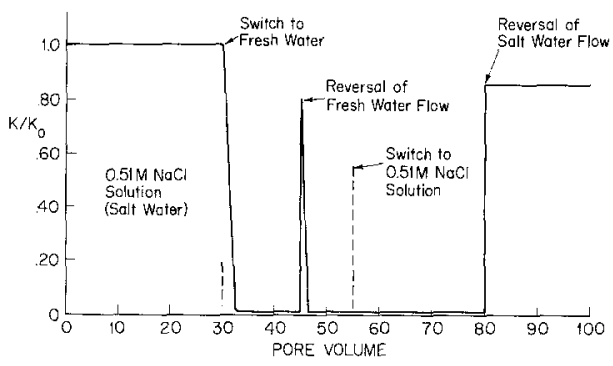

FIG. 1. Permeability reduction, temporary and permanent permeability gain in water sensitivity of Berea sandstone.

The study of the water sensitivity of sandstone is of practical importance to the oil and gas-producing industries since this is a potential problem in many field operations such as drilling, solution mining, acidization, and, in particular, water and polymer flooding. The injection of a fluid whose solution chemistry is not compatible with the porous rock can bring significant reductions in the formation permeability. Therefore, controlling the water sensitivity of sandstone is essential to economical and effective operation of petroleum reservoirs.

The study of water sensitivity began in 1943 , and up until 1964 it was believed that the swelling of montmorillonite and mixed-layer clays caused both pore blockage and the decrease in permeability. Only during recent years has clay particle migration been considered a factor. However, particle migration is now thought to be the most important damage mechanism in water sensitive sandstones (2), owing to the fact that damage occurs in sandstones containing no swelling clays.

The authors have developed a physical model describing the phenomenon (3). It was found that when flow is switched from salt water to fresh water, clay particles are released from the pore wall. The particles then migrate in the direction of the flow and are trapped at pore throats which results in blockage of the pores of the sandstone, thereby decreasing the permeability. The fact that the clay particles are released only when the fresh water replaces the salt water strongly indicates that the release process is dependent on the salt concentration. Yet, the previous basic studies (4-8) on this phenomenon primarily focused on trying to prevent the release of the particles by varying the $\mathrm{pH}$, the types of salts and polyelectrolytes present. Moreover, these studies, in general, were essentially of an exploratory nature.

This paper examines the phenomenon of water sensitivity of Berea sandstone in the presence of different salts as a function of salt concentration. The effects of various pertinent physical and chemical parameters on the peptization of the dispersable clay particles are presented and the results are discussed in terms of the existing double-layer theory.

\section{EXPERIMENTAL APPARATUS AND PROCEDURES}

A schematic diagram of the experimental apparatus is shown in Fig. 2. Using a twin cylinder Ruska pump fluid is forced through two Millipore submicron filters and the sandstone core. The filters trap suspended particles larger than $0.4 \mu \mathrm{m}$ in diameter. The fluid enters the core through a Plexiglas distributor (D) and an identical Plexiglas collector (E) is used to converge the fluid exiting the core. The collector and the distributor also provide support for the platinum electrodes which are used to measure the salt concentration. The pressure drop across the core is measured using transducers with various strain gauge adaptors. Both the pressure drop and the salt concen-

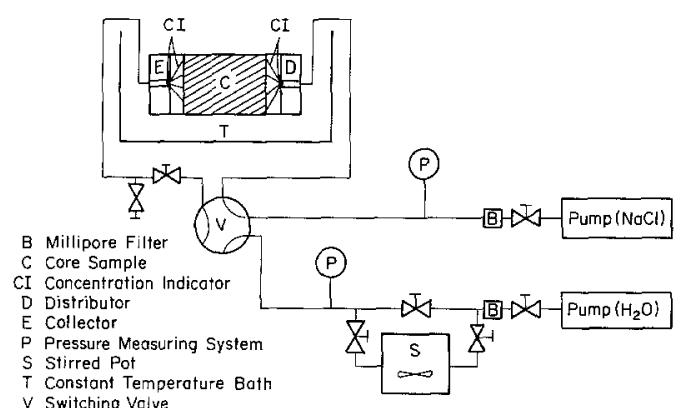

FiG. 2. Schematic diagram of the experimental set-up.

Journal of Colloid and Interface Science, Vol. 10L, No. 1, September 1984 
tration are continuously recorded with an Omniscribe dual channel strip chart recorder.

The sandstone core, the Plexiglas distributor, and the collector are placed inside a 2-in. Tygon tube and put into a Hassler core holder. The annular space between the tube and the core holder is filled with $90 \mathrm{~W}$ motor oil and pressurized by pre-purified $\mathrm{N}_{2}$ gas to prevent the fluid from flowing over the core surface. The core holder is then placed in a constant temperature bath. A more detailed description of the core holder, Plexiglas distributor, etc., is given elsewhere (1).

There were two reasons for the use of Berea sandstone. First, it contains about $8 \%$ by weight of dispersable clays, primarily kaolinite with some illite, $12 \%$ feldspar, and $80 \%$ quartz. No swelling clays (montmorillonite) were detected in any of the X-ray analyses and, therefore, Berea sandstone can be used for studies on clay dispersion in sandstone. Second, Berea sandstone is used as a reference sandstone in the oil-producing industries. It is estimated to be of Mississippian age on the geological time scale and is found in Berea, Ohio. The porosity of the sandstone core is $19 \%$.

In a typical experiment, a Berea sandstone core $1 \mathrm{in}$. in diameter and $1 \mathrm{in}$. in length was vacuum saturated for 2 to 3 days in a $0.51 \mathrm{M}$ ( $3 \mathrm{wt} \%$ ) salt solution. The saturated Berea core was then placed inside the core holder and a $0.51 M$ test salt solution was sent through the core, at a given superficial velocity. The salt concentration was then decreased in small steps, until a drop in sandstone permeability was observed. To verify that the sandstone core being used in the experiment was indeed water sensitive, an abrupt switch of the flow from salt water to fresh water was made on the last step of the run. The experiments were performed with different monovalent and divalent solutions. The $\mathrm{pH}$ of the solution in all cases were around neutral ( $\mathrm{pH} 7)$.

The permeability was computed appropriately by using Darcy's law for one dimensional horizontal laminar flow of the homogeneous fluid through a porous medium. In this particular case this is given by

Journal of Colloid and Interface Science. Vol. 101, No. 1, September 1984

$$
\frac{q}{A_{\mathrm{c}}}=V=\frac{-K}{\mu} \frac{\Delta P}{L}
$$

where

$q=$ volumetric flowrate

$A_{\mathrm{c}}=$ cross-sectional area of the medium

$\Delta P=$ pressure drop across the medium

$L=$ length of the medium

$\mu=$ viscosity of the fluid

$K=$ permeability

$V=$ superficial velocity of fluid.

\section{RESULTS}

\section{The Critical Salt Concentration}

The normalized core permeability, $K / K_{0}$, resulting from a stepwise decrease of the $\mathrm{NaCl}$ salt concentration at $30^{\circ} \mathrm{C}$ and at a superficial velocity of $19 \mathrm{~cm} / \mathrm{hr}$ is shown in Fig. 3. The dashed vertical line in the figure indicates the pore volume at which the abrupt change in salinity concentration was made to the fluid entering the core. For example, after 40 pore volumes of $30,000 \mathrm{ppm} \mathrm{NaCl}$ have passed through the core, the flow is switched so that a $10,000 \mathrm{ppm} \mathrm{NaCl}$ solution begins to flow through the core. One observes in Fig. 3 that there is no reduction in permeability $\left(K / K_{0}\right.$ $=1.0$ ) for a series of step decreases from 30,000 to $4250 \mathrm{ppm}$. Only when the salt concentration was reduced from a concentration of 4250 to $4000 \mathrm{ppm}$, the core permeability decreased by $43 \%$. These results show that the core permeability only decreases when the fluid in

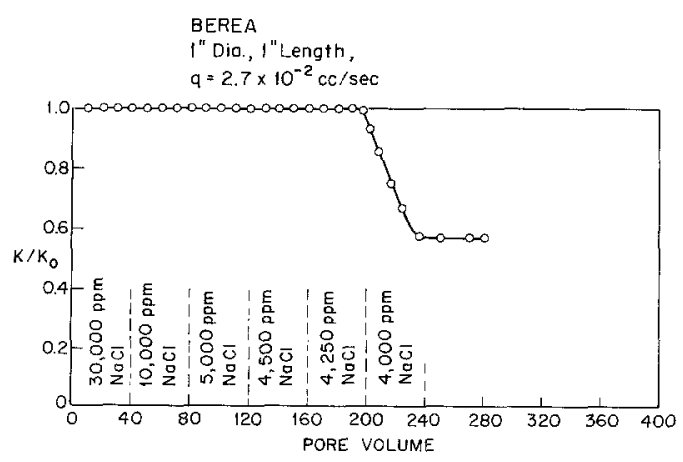

FIG. 3. Critical salt concentration in water sensitivity of Berea sandstone. 
contact with the core has a salt concentration below 4,125 ppm $( \pm 125)$.

The effluent samples of fluid collected at each salt concentration step were analyzed (1). It was found that the only sample that contained clay particles was, as expected, the one collected during the flow of $4000 \mathrm{ppm}$ solution. These particles were virtually all kaolinite with some traces of illite, as revealed by scanning electron microscope (SEM) analysis (1). Consequently, one concludes that the clay particles present in the sandstone core are not released until the salt concentration of the fluid in contact with the sandstone drops below a certain salt concentration. This salt concentration is defined as the "critical salt concentration" (CSC). The CSC for sodium chloride at $30^{\circ} \mathrm{C}$ and at a superficial velocity of 19.2 $\mathrm{cm} / \mathrm{hr}$ is $4125 \mathrm{ppm} \pm 125$, i.e., 0.070 $\pm 0.002 \mathrm{M}$.

The CSC is analogous to the critical flocculation concentration (CFC) in flocculation of colloidal particles (9). The critical flocculation concentration (CFC) for $\mathrm{Na}^{+}$for kaolinite suspensions (10) is of the order of $10^{-2}$ $M \mathrm{Na}^{+}$which is approximately $20 \%$ of the CSC for $\mathrm{Na}^{+}$. The $\mathrm{NaCl}$ concentration at which fine grain quartz particles begin to flocculate and sediment is found to be $0.06 \mathrm{M}$ $\mathrm{NaCl}(11)$, which is close to the CSC of 0.07 $M \mathrm{NaCl}$.

The CSC is a useful parameter in oil and gas production processes since one can attempt to avoid reduction in formation permeability by trying to prevent the formation to come in contact with an aqueous solution with a salt concentration below the CSC.

To examine the effect of various physical and chemical factors on the critical salt concentration, experiments were performed in two areas. In the first, the effects of parameters such as the flowrate and temperature on the CSC of the commonly used salt, sodium chloride, were studied. In the second area, the CSCs of various other salts, both monovalent (e.g., $\mathrm{LiCl}, \mathrm{CsCl}$ ), and bivalent (e.g., $\mathrm{CaCl}_{2}$ ), were investigated. The following summarizes the results.

\section{Effect of Superficial Velocity and Temperature on CSC}

The effects of superficial velocity on the CSC of sodium chloride were studied over a range of velocity of 3.2 to $570 \mathrm{~cm} / \mathrm{hr}$ at a temperature of $30^{\circ} \mathrm{C}$. It was found that the CSC is independent of velocity in this range (1). The highest velocity, $570 \mathrm{~cm} / \mathrm{hr}$ corresponds to some 500 times the typical linear velocity of $1 \mathrm{ft} /$ day realized in oil production.

The variation of the CSC with temperature was studied for $\mathrm{NaCl}$ solutions over the temperature range of 0 to $60^{\circ} \mathrm{C}$. A wider range was not possible due to the equipment limitations. Figures 4 and 5 show the variation in permeability as the salinity was decreased sequentially at constant temperatures of 0 and $60^{\circ} \mathrm{C}$, respectively. The values of the CSC for the temperatures studied are presented in Table I and it is observed that the CSC is directly dependent on the temperature.

\section{Critical Salt Concentration of Other Salts}

The critical salt concentrations (CSCs) of various other salts were obtained by conducting step-decrease experiments at $30^{\circ} \mathrm{C}$ and at a superficial velocity of $19.2 \mathrm{~cm} / \mathrm{hr}$.

The critical salt concentrations of $\mathrm{NaI}$ and $\mathrm{Na}_{2} \mathrm{CO}_{3}$ were found to be $0.069 M \pm 0.002$ and $0.035 M \pm 0.002(0.07 M$ with respect to $\mathrm{Na}^{+}$), respectively. These values of the CSC are in excellent agreement with that of $\mathrm{NaCl}$ and consequently one concludes that the anions $\mathrm{Cl}^{-}, \mathrm{I}^{-}$, and $\mathrm{CO}_{3}^{-2}$ have no effect on the
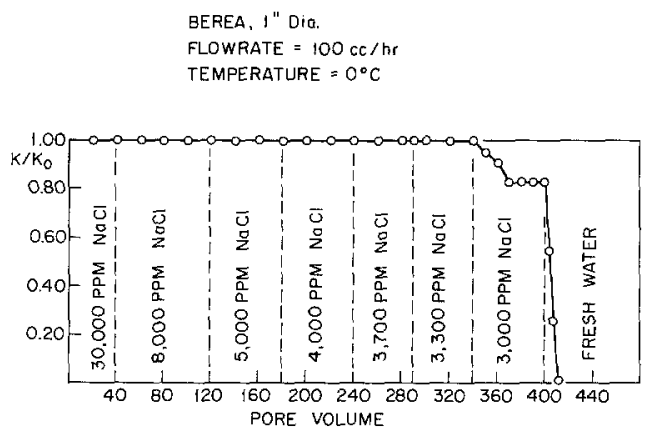

FIG. 4. Critical salt concentration at low temperature. 


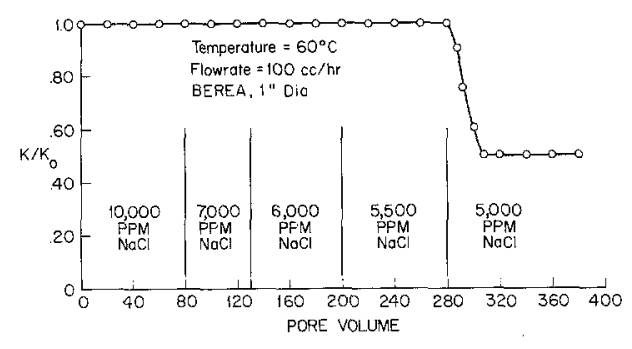

FIG. 5. Critical salt concentration at high temperature.

CSC, and therefore the CSC is primarily a characteristic of the cation. This finding is rather significant since it agrees qualitatively with the Schulze-Hardy rule (9).

Effect of charge of a cation on the CSC. The charge or valency of a cation was found to have a dramatic effect on the CSC. In fact, it was observed that the phenomenon of "water sensitivity" does not occur when bivalent salt solutions such as $\mathrm{CaCl}_{2}$ are used in place of sodium chloride solutions. Figure 6 shows the results of shock experiments $(0.51 \mathrm{M}$ salt solutions followed by fresh water) for a number of salt solutions. In a shock experiment the core is first vacuum saturated with the test salt solution and then a shock experiment as described earlier is conducted. The shock experiments for bivalent salt solutions were followed by resaturation of the core with a 0.51 $M$ sodium chloride solution. Fresh water was then sent through the core to complete the $\mathrm{NaCl} /$ water shock experiment using the same core as in the bivalent salt shock experiments. In all cases, a drastic and rapid reduction in permeability was observed indicating that all cores were indeed water sensitive. One ob-

TABLE I

The CSC as a Function of Temperature ${ }^{a}$

\begin{tabular}{cc}
\hline Temperature $\left({ }^{\circ} \mathrm{C}\right)$ & $\operatorname{CSC}$ (moles/liter) \\
\hline 0 & $0.056 \pm 0.002$ \\
30 & $0.070 \pm 0.002$ \\
60 & $0.090 \pm 0.004$ \\
\hline
\end{tabular}

${ }^{a}$ Velocity $=19.2 \mathrm{~cm} / \mathrm{hr}$.

Journal of Colloid and Interface Science, Vol. 101, No. 1, September 1984

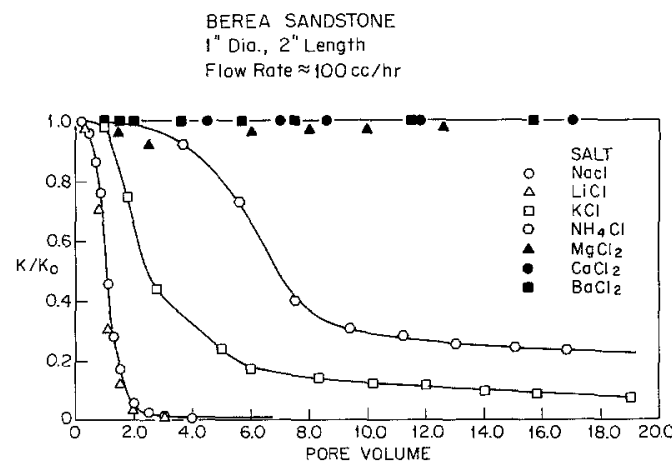

FIG. 6. Comparison of permeability reduction for various salt solutions.

serves in Fig. 6 that there are no reductions in the permeability in shock experiments using bivalent salt solutions with the exception of a $2 \%$ reduction in permeability for $\mathrm{MgCl}_{2}$.

Effect of monovalent cations on the CSC. The effect of the type of monovalent cation on the CSC was examined by conducting stepdecrease experiments at $30^{\circ} \mathrm{C}$ and at a velocity of $19.2 \mathrm{~cm} / \mathrm{hr}$. The CSCs for 5 different monovalent cations are listed in Table II. As can be seen in Table II, both similarities and dissimilarities were found among CSCs of various monovalent cations. While the CSCs for $\mathrm{Na}^{+}$and $\mathrm{Li}^{+}$are virtually the same at about $0.07 \mathrm{M}$, the CSCs for $\mathrm{NH}_{4}^{+}$and $\mathrm{Cs}^{+}$are significantly lower.

\section{DISCUSSION}

The peptization of clay particles in contact with various salt solutions resembles in some respects that of lyophobic colloids. The critical salt concentration (CSC) is analogous to the critical flocculation concentration (CFC), and that the CSC of the counterions decreases with increasing cation charge or valency. There are, however, some rather important differences between the clay system and a typical lyophobic solution. While the CSC is virtually nonexistent for divalent counterions, a CFC does exist for typical lyophobic solutions of divalent counterions. Furthermore, for clay dispersion the difference among the CSCs of monovalent cations are much greater and they 
TABLE II

CSC of Monovalent Cations

\begin{tabular}{rccccc}
\hline & & $\begin{array}{c}\text { Ionic } \\
\text { radius } \\
(12) \\
(\AA)\end{array}$ & $\begin{array}{c}\text { Hydration } \\
(12) \\
\text { (number } \\
\text { of moles) }\end{array}$ & $\begin{array}{c}\text { Hydrated } \\
\text { radius } \\
(12)(\AA)\end{array}$ \\
\hline $\mathrm{Na}^{+}$ & $0.07 \pm 0.002$ & 0.98 & 10 & 7.9 \\
$\mathrm{Li}^{+}$ & $0.068 \pm 0.002$ & 0.78 & 12 & 10.0 \\
$\mathrm{~K}^{+}$ & $0.044 \pm 0.003$ & 1.33 & 5 & 5.3 \\
$\mathrm{NH}^{+}$ & $0.013 \pm 0.005$ & 1.43 & 2 & 5.3 \\
$\mathrm{Cs}^{+}$ & $0.006 \pm 0.002$ & 1.65 & $<1$ & 5.0 \\
\hline
\end{tabular}

may vary by as much as an order of magnitude.

The double-layer theory has been used widely in colloidal studies to explain the coagulation and peptization of colloidal solutions. For example, by using the double-layer theory, Matijevic and co-workers $(13,14)$ were ble to describe the effect of $\mathrm{pH}$ and the electrolyte strength on the adsorption and desorption of submicronic chromium hydroxide particles on glass and steel plates. The qualitative features of some of the results of the present work can also be explained with the aid of this theory. The major forces that hold the clay particles to the porewalls are the London-van der Waals forces of attraction. The forces causing the detachment of the clay particles are the double-layer forces of repulsion and possibly, under conditions of high flowrate, the hydrodynamic shear. Calculations show these two forces of double-layer and hydrodynamic shear to be the most probable cause for particle release compared to a number of others which have been proposed such as osmotic shock (Mungan (5) has showed osmotic shock is not the cause of particle release).

Experimental observations strongly suggest that the double-layer force of repulsion may be the dominant cause of particle release. These observations include the effects of the two most important parameters that govern the stability of the colloidal suspension of clay particles: temperature and type of cation. More importantly, the phenomenon occurs only at relatively low salt concentrations where the double-layer repulsive forces are large. This fact also strongly supports the double-layer hypothesis of particles release. This work provides data on the CSC of various salt solutions considering the effects of the temperature and the flowrate. A theoretical analysis of these effects is presented in the next section.

\section{Analysis of the Effects of Temperature and Flowrate}

To carry out the double-layer theory analysis, the system of particle of radius $a_{\mathrm{p}}$ attached to the pore wall of a radius $r$ is considered to be a sphere attached to a flat surface $\left(a_{\mathfrak{p}} \ll r\right)$. A different particle geometry, that of platelets, has also been assumed in the double-layer calculation and found to give essentially the same results (1). Using the flatplate-sphere system, the effect of flowrate on the CSC was studied by comparing the forces due to flow with the London-van der Waals forces of attraction. The London-van der Waals force of attraction $F_{\mathrm{A}}$, can be represented by:

$$
F_{\mathrm{A}}=\frac{-A a_{\mathrm{p}}}{6 H^{2}} .
$$

It is assumed that (1) the particle radius is much greater than the distance of separation $H$, and (2) the retardation effect is negligible for $H$ of the order of $10 \AA$. In the above equation $A$ is the Hamaker's constant.

Hydrodynamic forces in the direction perpendicular to the flow $\left(F_{\mathrm{H}}\right)$ will exist if the particle is not spherical. It was assumed that the upper bound of this force is equal to that in the flow direction and is given by O'Neill (15):

$$
F_{\mathrm{H}}=32 a_{\mathrm{p}} U_{\mu}
$$

where $\mu$ is the viscosity of the liquid and the velocity, $U$ was taken as equal to the average interstitial velocity of the larger pores (4 to 10 $\mu \mathrm{m}$ in radius) which contribute to about $60 \%$ of the porosity of the Berea sandstone. The hydrodynamic force was computed at a velocity of $568 \mathrm{~cm} / \mathrm{hr}$ which is the highest velocity used in our experiments. It was found 
(1) that even at this high velocity, which is about 3 orders of magnitude higher than typical velocities used in the oil and gas production industries, the hydrodynamic force is negligible compared to the London-van der Waals attractive force computed over the typical range of Hamaker's constant from 5 $\times 10^{-14}$ to $5 \times 10^{-13}$ erg. Consequently, we conclude that the superficial velocity does not affect the CSC and this prediction was verified experimentally.

In order to study the effect of temperature, the CSC was treated in the same manner as the critical flocculation concentration (CFC) in the Derjaguin-Landau-Verwey-Overbeek theory (DLVO) (9). The CSC is defined as the salt concentration below which permeability reduction and therefore particle release takes place. Mathematically, the particle release is assumed to occur at the point for which both the total energy $V_{\mathrm{T}}$ and the total force $F_{\mathrm{T}}$ acting on the clay particle attached to the pore wall are equal to zero. Neglecting the thermal energy of particles and the hydrodynamic force, therefore, one has

$$
\begin{aligned}
& V_{\mathrm{T}}=V_{\mathrm{R}}+V_{\mathrm{A}}=0 \\
& F_{\mathrm{T}}=\frac{-d V_{\mathrm{T}}}{d H}=F_{\mathrm{R}}+F_{\mathrm{A}}=0
\end{aligned}
$$

where the subscript $R$ stands for the doublelayer repulsion and $\mathrm{A}$ for the London-van der Waals attraction.

Williams and Williams (16) have reported that the face zeta potential of kaolinite decreased from -33 to $-39 \mathrm{mV}$ for a 100 -fold variation of $\mathrm{NaCl}$ concentration from $10^{-2}$ to $10^{-4} \mathrm{M}$ while the edge zeta potential remained zero for nearly neutral $\mathrm{pH}$ conditions (the $\mathrm{pH}$ condition in the present work). While the face potential in this particular case can be taken as $-30 \mathrm{mV}$, the variations of both face and edge potentials with $\mathrm{pH}$ should not be ignored if the $\mathrm{pH}$ is substantially different from a neutral $\mathrm{pH}$, such as the case of sandstones containing considerable amount of carbonates. Such low variation in zeta potential indicates that the system can be modeled as having a constant surface potential in computing the double-layer interaction. Furthermore, it was assumed that the wall and the particle are of the same potential which follows since the streaming potential of larger pores do not differ significantly from the zeta potential of the particles. The interaction energy, $V_{R}$ for a sphere-plate system is given by the expression (Honig and Mul (17)):

$$
\begin{aligned}
V_{\mathrm{R}}= & \frac{128 \pi n k T}{\kappa^{2}}\left[\gamma^{2}\left(1+2 / 3 \gamma^{2}\right) \ln \left(1+e^{-\kappa H}\right)\right. \\
& +\frac{\gamma^{4}}{6}(\kappa H \tanh (\kappa H / 2)-\kappa H \\
& +\frac{\kappa H \tanh (\kappa H / 2)}{2 \cosh ^{2}(\kappa H / 2)} \\
& \left.\left.\quad-\frac{1}{\cosh ^{2}(\kappa H / 2)}+\cdots\right)\right] a_{\mathrm{p}}
\end{aligned}
$$

where

$$
\begin{gathered}
\gamma=\tanh \frac{\left(Z_{\infty}\right)}{4}, \quad Z_{\infty}=\frac{\nu e \psi_{\infty}}{k T}, \\
\kappa=\left(\frac{8 \pi e^{2} \nu^{2} n}{\epsilon k T}\right)^{0.5}
\end{gathered}
$$

which is valid for $\kappa H>0.75$ and $Z_{\infty}<5$.

Equations [2]-[5] were solved simultaneously to yield

$$
\kappa H_{m}=1.12
$$

which is the criterion for the release of clay particles according to the assumed model. This criterion together with Eq. [3] or Eq. [4] enable the evaluation of the Hamaker's constant for the system, A, and the distance of separation, $H_{\mathrm{m}}$, provided the CSC at a particular temperature is known. The CSC of $0.071 \mathrm{M}$ at $30^{\circ} \mathrm{C}$ was used in this calculation. The $\mathrm{Ha}$ maker's constant and the distance of separation were found to be $1.62 \times 10^{-13} \mathrm{erg}$ and $13 \AA$, respectively. The value of Hamaker's constant is within the range of $3 \times 10^{-14}$ to $6 \times 10^{-13}$ ergs for the silica-water system (18), and the value of $H_{\mathrm{m}}$ is reasonable (19). The values $\mathrm{A}$ and $H_{\mathrm{m}}$ are independent of temperature in the temperature range 0 to $60^{\circ} \mathrm{C}$. Consequently, with the knowledge of $\mathrm{A}$ and 
$H_{\mathrm{m}}$, one can compute the CSC at other temperatures by solving Eqs. [3] and [4] simultaneously. The CSCs at 0 and $60^{\circ} \mathrm{C}$ were found to be 0.063 and $0.079 M$, respectively. These computed values are in reasonable agreement with experimental values of $0.056 M \pm 0.002$ and $0.090 M \pm 0.004$. This analysis supplements the double-layer hypothesis for particle release as well as providing a rationale for the existence of the CSC. The total potential energy $\left(V_{\mathrm{T}}\right)$ plot can be used to illustrate the conditions for particle release. As shown in Fig. 7, at a salt concentration of $0.20 \mathrm{M}$ which is above the CSC of $0.07 M$, the total potential energy is negative and, therefore, the particles adhere to the pore wall. At a salt concentration below CSC, particles are expected to desorb from the pore wall provided that they overcome the energy barrier. It is realized that the particles are slightly separated from the pore wall due to the spreading pressure of the adsorbed electrolyte (20). This distance of closest approach $H_{0}$ is estimated to be of the order of hydrated size of $\mathrm{Na}^{+}$ion $(8 \AA)$ and at such a separation the difference between the potential energy and the maximum would be rather small. Therefore at a salt concentration of $0.02 \mathrm{M}$, which is below the CSC, the particles are released from the pore wall. The total interaction energy $\left(V_{\mathrm{T}}\right)$ at the location of maximum is comparable to that obtained by Kuo and Matijevic (14).

\section{The Effect of Cationic Charge}

Experimentally, it was found that the valence of a counterion has a dramatic effect on the clay particles release phenomenon with the CSC decreasing to become virtually nonexistant for bivalent salt solutions. A similar trend is observed in the data of Swartzen-Allen and Matijevic (10). They report the CFC for kaolinite particles in the solutions of $\mathrm{Na}^{+}$and $\mathrm{Ca}^{2+}(\mathrm{pH} \mathrm{7})$ to be respectively $1.6 \times 10^{-2}$ and $2.5 \times 10^{-4} \mathrm{M}$. The variation of the CSC with the cationic charge also agrees qualitatively with the DLVO theory.

The fact that the CSC is nonexistant for

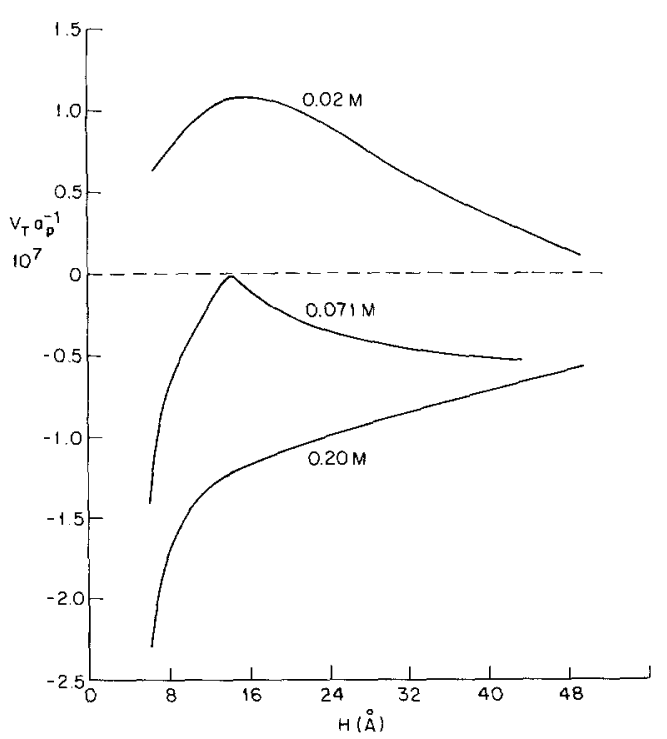

FIG. 7. Calculated total potential energy $\left(V_{\mathrm{T}}\right)$ as a function of distance of separation $(H)$. (For $a_{\mathrm{p}} \simeq 1 \mu \mathrm{m}$, the vertical scale is $1 \simeq 240 \mathrm{kT}$ at $30^{\circ} \mathrm{C}$.)

bivalent cations bears some analogy to the widely known repeptization phenomenon (21, 22). It has been observed that repetization is relatively easy when a solution contains monovalent counterions. However, the repeptization is effectively prevented when polyvalent counterions are present in a solution and this has been attributed to the difficulty of expelling them from the doublelayer. The same reasoning can be shown to explain the behaviour presented in this work (Fig. 6) by analyzing the following two experiments.

In one $\mathrm{NaCl}$-water shock experiment, fresh water continued to flow through the core until the concentration of $\mathrm{Na}^{+}$in the core effluent dropped to below 1 ppm. A $5 \mathrm{wt} \% \mathrm{CaCl}_{2}$ solution was then passed through the core to displace any $\mathrm{Na}^{+}$ions, if present, in the core. No $\mathrm{Na}^{+}$ions were detected in the effluent $\mathrm{CaCl}_{2}$ solution, thereby showing that all the $\mathrm{Na}^{+}$ions had been displaced and carried away by the fresh water flow during the shock.

In the second experiment, a $\mathrm{CaCl}_{2}$-water shock was produced by using a $\mathrm{CaCl}_{2}$ saturated core and following the same procedure as in 
BEREA

I"Dia., I"Length

$100 \mathrm{cc} / \mathrm{hr}$

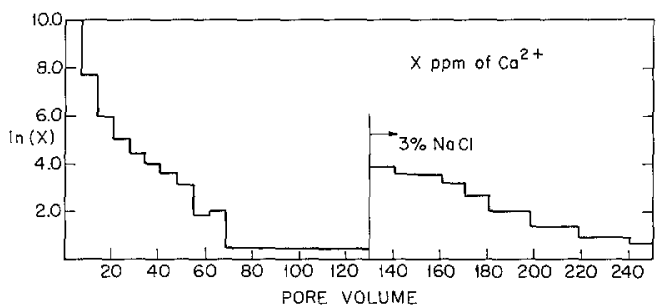

FIG. 8. Concentration of calcium ions during fresh water and salt water flow.

the previous experiment. Next, a $3 \mathrm{wt} \% \mathrm{NaCl}$ solution was passed through the core to displace the $\mathrm{Ca}^{2+}$ ions remaining in the doublelayers surrounding the clay particles in the sandstone core. The amount of $\mathrm{Ca}^{2+}$ ions displaced during the flow of sodium chloride solution can be found from the area under the curve shown in Fig. 8. This amount was equal to $0.22 \mathrm{meq}$, which is in good agreement with the measured cation exchange capacity (CEC) of the used core of 0.30 meq.

This observation can be explained by analyzing the variation of distribution coefficient in sorption of counterions of higher valences on a charged surface (exchanger). The distribution coefficient increases with increasing valences of counterions (23). A pertinent result is that the distribution coefficient of polyvalent ions increases very rapidly as the concentration of ions in the bulk solution decreases. These counterion valence effects are termed as electro-selectivity and can be explained in terms of Donnan potential (23). Applying these results to sandstone water sensitivity when the flow is switched from $\mathrm{CaCl}_{2}$ solution to fresh water and thus the concentration of $\mathrm{Ca}^{2+}$ ions is made to decrease, the distribution coefficient of $\mathrm{Ca}^{2+}$ increases significantly. Consequently, a sufficient amount of $\mathrm{Ca}^{2+}$ ions remains adsorbed onto the clay surface, even after a long exposure to the permeating fresh water.

\section{Specific Effects of Monovalent Cations}

Inspection of the CSC values reported in Table II reveals that there are significant dif- ferences among different monovalent cations. For example the CSC for $\mathrm{Cs}^{+}$is smaller than that for $\mathrm{Na}^{+}$by more than an order of magnitude. Swartzen-Allen and Matijevic (10) also report the same order of magnitude difference in the CFC of montomorillonite clay particles in solutions of $\mathrm{Cs}^{+}$and $\mathrm{Na}^{+}$cations. As the data in Table II show, the CSC of monovalent cations follows the sequence of

$$
\mathrm{Na}^{+}>\mathrm{K}^{+}>\mathrm{NH}_{4}^{+}>\mathrm{Cs}^{+}
$$

with $\mathrm{Li}^{+}$to be almost identical to $\mathrm{Na}^{+}$. This CSC trend is the same as that for CFC for negatively charged suspensions (9) and is also the same as the trend for adhesive forces observed for quartz and glass spheres adhering to a glass surface (11).

This CSC sequence may be compared with the adsorbability sequence of the special cations on the surface. In general, the specific adsorption of an ion is enhanced by large size and by lower hydration which itself is a function of ion size. As Table II shows, among the cations listed above, the large $\mathrm{Cs}^{+}$ion is the most strongly adsorbed and the small but highly hydrated $\mathrm{Na}^{+}$ion is adsorbed least.

The differences among the CSCs of monovalent cations may be related to their adsorption characteristics. As the salt concentration in the solution decreases the number of sites on the clay surface which are occupied by the monovalent cation decreases. At a given solution concentration, however, a greater amount of cation will remain on the clay surface for a strongly adsorbed cation since they are the most difficult to be displaced (12). As a result, a lower solution concentration has

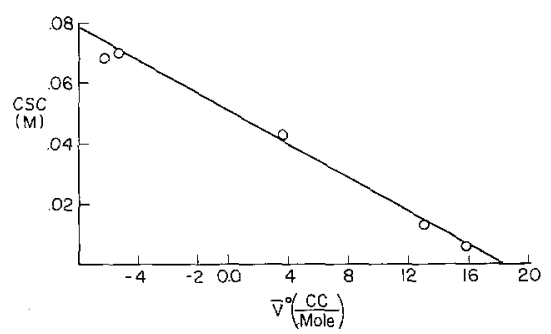

FIG. 9. Variation of critical salt concentration with ionic partial molar volume of monovalent cations. 
to be reached before the clay particles start to release from the pore walls (lower CSC). In addition, a strongly adsorbed counterion is more effective in reducing the surface potential (via the stern region) of clay particles, and therefore would result in a lower CSC. The CSC of monovalent cations is empirically found to correlate linearly with the ionic partial molar volume of the cation, $\dot{V}$ as shown in Fig. 9.

The hydration hypothesis is consistent with the results of a shock experiment where methanol was used in place of fresh water. Methanol being less polar than fresh water will not solvate the cations as much as water. Therefore, it should be difficult to displace the cations from the double-layers of clay particles with methanol which, in turn, should result in less dispersion of clay particles. As a result, the core permeability would not be reduced as much.

To test this hydration hypothesis, a solution of $(0.11 M) \mathrm{NaCl}$ in methanol was passed through the core until a constant pressure drop was obtained. The flow was then switched to pure methanol instead of fresh water as in the previous runs. Next, a $0.11 \mathrm{M}$ aqueous $\mathrm{NaCl} /$ water shock was conducted, using the same core. A $0.8 \mathrm{wt} \%(0.11 M) \mathrm{NaCl} /$ methanol solution was chosen owing to solubility considerations. The permeability reduction produced by the two different solvents is shown in Fig. 10. As one observes in this figure, the final reduction in permeability is less than $20 \%$ in the case of methanol shock, which is essentially negligible when compared with the 100 -fold reduction in permeability occurring in fresh water shock. Consequently it is seen that when the displacement of cations is suppressed due to inhibition of hydration by using a less polar solvent, the reduction in permeability is less. Such a finding demonstrates the importance of the hydration characteristics of cations in the water sensitivity of sandstone.

\section{SUMMARY}

It was found that a critical salt concentration (CSC) exists in the phenomenon of water sen-

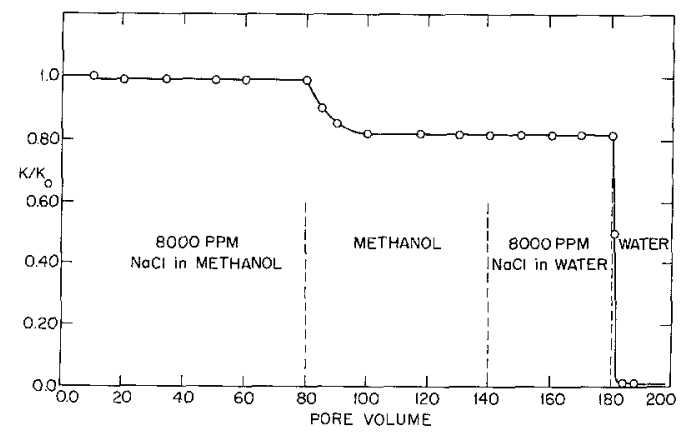

FIG. 10. Comparison of permeability reduction in methanol shock and water shock.

sitivity. Above the CSC no reduction in core permeability occurs with change in salinity. The CSC for sodium chloride solution was found to be $0.071 \mathrm{M}$. The CSC was found to be independent of flowrate in the range studied, but decreased with decreasing temperature. An analysis based on a force and potential energy balance on a clay particle attached to the pore wall was used to describe the effects of flowrate and temperature.

The critical salt concentration was found to be strongly dependent on the nature of cations. The CSC was found to be practically absent in the case of bivalent cations, while it varied significantly with the type of monovalent cations. The cation effect can be explained in terms of the degree of difficulty of expelling a cation from the clay surface. The difficulty of expulsion can be qualitatively related to the charge and the hydration of cations, while the charge effects predominate over the hydration effects. Without considering the electroselectivity effects, that strongly bind the cations on the clay surface, the simple DLVO theory cannot account for the variation of CSC with the type and the charge of counterions.

\section{ACKNOWLEDGMENTS}

The authors appreciate the support provided by the National Science Foundation Grant NSF-G-ENG7906647, the assistance in the manuscript preparation by S. F. Kia and J. S. Ahluwalia, and discussions with M. G. Reed and R. Schmidt of Chevron Oil Field Research and with the late Professor T. S. Burn who fostered one of the author's (HSF) initial interest in this subject while 
on sabbatical at the University of Bergen, Bergen, Norway. In addition, the useful suggestions of one of the referees is greatly acknowledged.

\section{REFERENCES}

1. Khilar, K. C., Ph.D. Thesis, University of Michigan (1981).

2. Gray, D. H., and Rex, R. W. 14th National Conference on Clays and Clay Minerals, 335 (1966).

3. Khilar, K. C., and Fogler, H. S., Soc. Pet. Eng. J. 23, 55 (1983).

4. Mungan, N., J. Pet. Tech. 17, 1449 (1965).

5. Mungan, N., J. Pet. Tech. 20, 113 (1968).

6. Jones, F. O., Jr., J. Pet. Tech. 16, 441 (1964).

7. Valey, C. D., J. Pet. Tech. 21, 1112 (1969).

8. Reed, M. G., J. Pet. Tech. 24, 869 (1972).

9. Hiemanz, P. C., "Principles of Colloid and Surface Chemistry." Marcel Dekker, New York, 1976.

10. Swartzen-Allen, S. L., and Matijevic, E., J. Colloid Interface Sci. 56, 169 (1976).

11. Read, A. C., and Hollick, C. T., Mat. Sci. Eng. 8, 202 (1976).
12. Grim, R. E., "Clay Mineralogy." McGraw-Hill, New York, 1968.

13. Kolakowski, J. E., and Matijevic, E., J. Chem. Soc. Faraday Trans. 1 75, 65 (1979).

14. Kuo, R. J., and Matijevic, E., ibid 75, 2014 (1979).

15. O'Neill, M. E., Chem. Eng. Sci. 23, 1293 (1968).

16. Williams, D. J. A., and Williams, K. P., J. Colloid Interface Sci. 65, 79 (1978).

17. Honig, E. P., and Mul, P. M., J. Colloid Interface Sci. 36, 258 (1971).

18. Lyklema, J., Adv. Colloid Interface Sci. 2, 65 (1968).

19. Visser, J., in "Surface and Colloid Science" (E. Matijevic, Ed.), Vol. 8, p. 3. J. Wiley, New York, 1976.

20. Lange, H., ACS Symp. Ser. Adsorption Interfaces 270 (1975).

21. Overbeek, J. Th.G., in "Colloid Science" (H. R. Kruyt, Ed.). Elsevier, Amsterdam, 1952.

22. Frans, G., and Overbeek, J. Th.G., J. Colloid Interface Sci. 38, 376 (1972).

23. Helfferich, F., "Ion Exchange." McGraw-Hill, New York, 1962. 